\title{
OBSERVATION S
}

SUR LE PROJET DE L'ALIÉNATION:

\section{DES FORETTS NATIONALES;}

PAR ÉTIENAE CUNIN,

\section{MEMBRE DU COMITÉ DES DOMAINES,}

Député du Département de la Meurthe.

Case

$278+2$

L'EUROPE a les yeux fixés fur la conduite de l'Affemblée nationale, elle pèfe, elle examine fes décrets; \& par celui que l'Affemblée rendra fur la queftion importante, foumife ǎ la difcuffion, 'l'Europe jugera fi la nation françaife eft en état de fe gouverner elle-même.

La confervation des forèts, entre les mains du gouvernement, jugée indifpenfable, par Sulli, Colbert \& l'Affemblée conftituante, qui, pendant tout le cours de fa feffion, en avoit pefé l'importance, ne paroiffoit plus devoir être mife en queftion. La perte irréparable que faifoit l'état en aliénant fes bois, eft fi

Domaines nationaux. I.

A 
clairement démontrée dans l'excellent rapport de $M$. Barrere, que, pourlesprincipes généraux, il me fuffira d'y renvoyer ceux qui ofent les combattre, \& qui n'y répondront jamais d'une manière fatisfaifante.

La propofition feule de l'aliénation eft impolitique, elle a jeté l'alarme dans tous les efprits, provoqué les réclamations d'une foule de départemens, \& confirmé les bruits femés par les émigrans, du délabrement total de nos finances.

Dańs la réuñión des membres des cinq comités, auxquels l'Affemblée a renvoyé l'examen de la queftion, il m'a paru que les partifans de l'aliénation étoient principalement des départemens méridionaux ou maritimes; les premiers à raifon de l'aridité de leur fol, ont peu de forêts \& point de futaie; n'ayant prefque point d'hiver, ils n'ont befoin de bois que pour la cuiffon de leurs alimens. Les départemens maritimes ont la reffource de la navigation, qui peut leur importer des bois de l'étranger; cette reffource leur fait prendre moins d'intérêt à la confervation des forêts nationales.

Mais, pour la majorité des départemens de l'intérieur, \& fur-tout pour ceux de la frontière d'Allemagne, du Luxenibourg \& de la Suiffe, j’ofe dire qu'en général l'aliénation des forêts feroit égálement deftructive des bois, du commerce, de l'agriculture, de la population, préjudiciable àlamarine, peu profitable à l'état, \& peut-êtreimpoffible à exécuter; c'eft ce que j'établirai après avoir relevé les erreurs dans lefquelles font tombés les partifans de l'aliénation.

Quoique je fente que cet écrit n'intéreffera, ni par le fuiet qui eft aride, ni par la diction pour laquelle je n'ai point de talens, prévoyant que n'etant pas infcrit des premiers pour la difcuffion, je ne pourrai obtenir la parole, jai cru devoir faire part à mes col- 


\section{(3)}

lègues, d'obfervations que la connoiffance du local, \& un peu d'expérience dans cette partie m'ont mis à portée de faire, \& je les prie de me lire avec patience.

La modeftie du premier orateur, partifan de l'aliénation, donne un nouveau mérite à fon travail; il a développé dans la première partie des connoiffances \& de bonnes vues fur l'ordre à introduire dans le mode de réferve \& d'exploitation des forêts; il a remarqué, avec raifon, que les maffifs de futaie ne pouvoient produire de bons bois de conftruction, \& qu'on n'en trouve qui réuniffent la folidité \& le nerf néceffaire, que dans les futaies fur taillis.

L'orateur eft forti fouvent de l'état de la queftion; il a accumulé à fon avis fur l'aliénation des forêts une digreffion fur leur organifation, fur le defféchement des marais \& le défrichement des landes : il n'a pas réféchi que ce font des queftions féparées, qui demandent chacuine fa difcuffion, \& dont la première feule étoit à l'ordre du jour; que s'il y a des vices dans l'organifation \& police des forêts, il faut les reetifier, mais que ces vices ne font point un motif fuffifant pour aliéner cette grande propriété nationale.

Cet orateur eft tombé dans de grandes erreurs, fur la confiftance de nos forêts, leur produit, leur valeur réelle, \& les frais de leur adminiftration; il a beaucoup diminué les uns, \& exagéré les autres. Qu'il me permette de lui obferver qu'il eft tombé dans la même faute que le père Petau, dans la differtation fur la multiplication du genre humain avant le déluge, \& qu'on peut lui répondre, comme à cet auteur, que les hommes, non plus que les madriers, ne fe font point avec la plume.

L'orateur eft tombé dans une inconféquence fenA 2 


\section{(4)}

fible; il a argumenté des frąis de l'adminiftration des forêts pour en induire, que pour fe fouftraire à cette dépenfe, il falloit les aliéner : d'un autre côté il eft convenu qu'on n'en devoit pas laiffer la difpofition illimitée aux acquéreurs; il faudra donc toujours une adminiftration pour les furveiller; la nation, par la vente de fes bois, ne fe fouftrairoit donc pas à cette dépenfe.

II a propofé d'effarter \& mettre en culture toutes les forêts fituées dans les plaines; il a préfenté une perfpective riante d'accroiffement de moiffons \& de pâturáges : fi les bleds \& le bétail étoient les feules chofes néceffaires à la vie, leur augmentation pourroit auffi me féduire; mais le bois étant d'une auffi abfolue néceffité que les vivres \& le vêtement, il y auroit de l'imprudence d'augmenter les feconds aux dépens des premiers, également indifpenfables. Il n'a pas réfiéchi que, fuivant la remarque des compagnies \& des individus les plus inftruits dans cette partie, en divifant la maffe des coupes réglées de l'intégrité de nos forêts, entre tous les habitans du royaume, chacun n'avoit pour fon chauffage, que le produit d'un trente-huitic̀me d'arpent, qui ne donne qu'un cinquiène de corde; or, cette quotité eft fi évidemment infuffifante, qu'il faut chercher à l'augmenter plutôt qu'à la réduire.

On objecte que la Provence \& le Languedoc, à raiion de la douceur de leur température, confomment peu de bois de chauffage; que la Flandre, l'Artois \& le Forez ne brûlent que de la tourbe ou du charbon de terre.

J'en conviens; mais on doit avouer également que les falines, forges, fonderies, \& autres ufines à feu, rempliffent au moins le vuide de la non-confommation 
des départemens de ces provinces; il s'enfuit donc toujours que la portion de chauffage de chaque habitant ne fera que d'un cinquième de corde.

On a exagéré le prix que la nation tireroit de la vente de fes forêts; on en a évalué l'arpent à 500 liv. On n'a pas réféchi que quatre millions d'arpens de plus, livrés à l'aliénation, feroient énormément baiffer le prix de tous nos domaines; que l'arpent de bois qui, dans uné infinité de cantons ne vaut pas so liv., fe portera tout au plus, le fort portant le foible, à 200 livres; que conféquemment le produit de la vente du tout ne produira pas 600 millions.

Mais fur quels motifs, fuivant fes partifans, cette aliénation feroit-elle fondée? fur le pea de produit des forêts qu'on dit ne pas fe porter annụellement à plus de 20 millions; fur les frais de leur adminiftration que l'on élève à près de 4 millions par an; fur l'impoffibilité phyfique que l'adminiftiation, comme elle eft organifée, puiffe fuffire à fes fonctions; fur la fuppofition que les forêts entre les mains des particuliers, produiront toujours plus qu'en celles de la nation; enfin, fur l'état-de nos finances, qui exige impérieufement la vente de nos forêts.

Je démontrerai fur le premier motif, que Pefprit de fyftême \& de calcul a fait tomber les auteurs de cette affertion dans des contradictions, des exagérations d'une part \& des réductions imaginaires de l'autre, qui détruifent leur propre fyftême ; fur le fecond, que les frais d'adminiftration beaucoup exagérés même dansle mode actuel, peuvent, fans nuire au bien du fervice, fe réduire à près de moitié moins de la fommeà laquelle on les porte; fur le troifième, que le nombre des adminiftrateurs foreftiers créés par l'affemblée conftitu-, ante, eft fuffifant pour leursfonctions, \& qu'il eft très-fa-

A 3 


\section{$(6)$}

cile de les leur faire exécuter; fứr le quatrième, que l'expérience \& les faits prouvent qu'en général il n'y a point de forêts plus mal tenues que celles des particuliers; fur le cinquième enfin, qu'il n'eft nullement prouvé que l'état de nos, finances exige la vente de nos forêts; mais que quand on fuppoferoit le fait vrai, ce feroit également nuire à la vente des biens nationaux \& à celle des forêts, que d'en propofer cumulativement l'aliénation.

\section{Réfutation du premier motif.}

Son premier auteur, après avoir affuré que le revenu de nos forêts eft tout au plus de 20 millions, avance, page 17 de fon opinion imprimée, que les feules fapinières des ci-devant provinces de Lorraine, Alface \& Franche-Comté, produiront annuellement I 20 millions de pieds cubes de bois'de conftruction: il ajoute cependant que l'effence qui domine dans les forêts de cés provinces eft le chêne ; d'où l'on doit conclure que le chêne de ces forêts doit produire au moins autant de bois de conftruction; les futaies réunies de ces trois provinces donneroient donc annuellement, fuivant fon calcul, 240 millions de pieds cubes de bois de conftruction.

Or, fuppofons que le pied cube de ce bois ne fe vende que 20 fous, ce qui eft certainement bien audeffous de fon prix réel, les feules futaies des forêts de ces trois provinces qui ne contiennent pas moitié des forêts du royaume, produiroient annuellement 240 millions; que l'autre moitié devant produire au moins la même fomme, parce que fi ce bois eft plus rare dans les autres parties du royaume, il doit néceffairement y hauffer de prix, le revenu des feules futaies de nos forêts s'élèveroit à 480 millions. Leur 
produit annuel en bois de chauffage, non compris les fagots, eft eftimé à 5,240,272 cordes; je fuppofo le prix de la corde à 6 livres, qui eft certainement le prix commun le plus bas, cette quantité de cordes donneroit un revenu de $30,44 \mathrm{r}, 632$ livres, qui ajouté aux 480 millions produit des feules futaies, donneroit un revenu de $510,441,632$ liv.

Il paffe pour conftant que le royaume contient treize millions cent mille arpens de forêts ; les partifans de l'aliénation fuppofent que la nation eft propriétaire de quatre millions de ces arpens, conféquemment des quatre treizièmes de la totalité des forêts du royaume : or, en divifant dans cette proportion les $510,441,632$ livires, à laquelle fomme, fuivant le calcul des partifans de la vente des forêts, s'élèveroit le produit annuel de toutes celles de France, il s'enfuivroit que la nation, pour les quatre treizièmesquiluiap. partiennent, percevroit annuellement I 30,058,960 liv: \& ce font les auteurs de ce caleul qui dans le même travail eftiment à 20 millions au plus le revenu des forêts nationales! une pareille contradiction démontre évidemment combien on doit fe défier de l'efprit de fyftême.

Quant à moi, qui détefte l'exagération, j’avancerai feulement que nos forêts peuvent produire dès-à-préfent un revenu de 25 millions qui, avec une bonne adminiftration, s'élèvera facilement jufques à 4 o.

Les partifans de l'aliénation portent à $3,885,8,30$ liv. les frais d'adminiftration de nos forêts: il eft évident qu'ils ne fe portent pas à beaucoup près à cette fomme; que moyennant quelques réformations peu fenfibles dans Porganifation foreftière, ils fe réduiront à près de moitié moins; je préfenterai même à la nation un projet qui fera abfolument tomber ces $A_{4}$ 


\section{(8)}

frais fur le luxe, fans qu'il en coûte rien à la maffe du peuple.

Je difcuterai d'abord les articles du tableau des dépenfes préfenté par les partifans de l'aliénation.

Les premier \& fecond articles portent à 50,000 liv. les gages des cinq commiffaires \& les frais de leurs tournées. J'avoue que fuivant le plan de l'organisation forestière, cette dépense se porteroit àpeu-près à la fomme tirée hors ligne; mais comme je maintiens que les cinq commiffaires doivent être réduits à un, \& que leurs tournées feroient parfaitement inutiles, je réduis les deux articles à $12,00 d$ livres pour les gages d'un seul commiffaire; la dépense de la nation fera donc, fur ces deux articles, diminuée de 38,000 liv.

J'ai dit que les cinq commiffaires doivent être réduits à un. Vous vous rappelez, Meffieurs, que fous l'ancien régime l'intendance de l'univerfalité des domaines \& bois nationaux étoit confiée à un feul homme. Or, fi fous ce régime diffipateur on avoit éprouvé qu'un feul fuffifoit à ce travail, il eft bien furprenant que l'Affemblée conftituante, çui avoit diftrait les domaines de cette partie de l'adminiftration, ait imaginé d'établir cinq perfonnes pour la police gé. nérale des feules forêts. Les deux premiers articles de dépenfe du tableau doivent donc être modérés au moins à 12,0001 . ci. Les art. 3 \& 4 qui fe portent à 78,8001 . 12,0001 . doivent être réduits à $32,000 \mathrm{l}$. les frais des bureaux des domaines \& bois réunis ne fe portoient qu'à cette dernière formme: or, le travail de ces bureaux

$12,0001$. 
D'autre part... $\cdots$ n'étant point augmenté, il y auroit de l'inconféquence à accroître fes falaires; les articles $3 \& 4$ doivent donc être modérés à 32,000 liv. ci. . . . .

Les articles $5 \& 6$ pour le bureau des plans \& artiftes, \& l'hôtel d'adminiftration que l'on porte enfemble à 32,000 liv. de dépenfe, en doivent être rayés entièrement; il exifte des plans \& cartes de toutes les forêts n\&tionales, tant dans les bureaux des grands maîtres que dans ceux du cidevant intendant des domaines; il eft donc fuperfiu d'employer des artiftes pour en faire d'autres: quant à l'hôtel d'administration, la dépense de fa location eft purement négative. Cet hôtel exifte \& appartient à la nation, il ne lui en coûtera donc rien pour fé le procurer.

L'art. 7 pour frais d'établiffement, port de lettres, regiftres \& marteaux que l'on porte annuellement à 20.000 liv. doit être réduità 2,000 1. Les frais d'établiffement ne, coûteront rien, puifque les bureaux font établis; les ports de lettres font une dépenfe négative; la dépenfe des marteaux ne fe fera qu'une fois dans vingt ans, \& ne peut corféquemment être regardée comme annuelle; la fourniture des regiftres est la feule qui

32,0001

12,0001
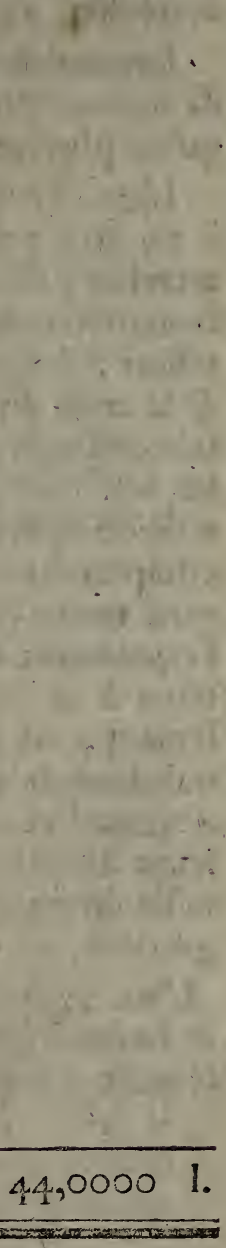


\section{(10)}

Ci-contre. . . . . . .

doive fe renouveler tous les ans: mais comme la nation ne paye que le papier \& non le timbre, cet article fe portera au plus à 2,000 liv. de dépenfe annuelle, ci . .......

Les articles 8, 9 \& 10 font juftes, ils doivent être paffés pour la fomme qu'ils portent, ci. . . .....

L'art. I I eft une erreur manifefte, il ne doit point $y$ avoir d'arpenteurs attachés à chaque infpection, mais feulement un petit nombre à chaque divifion, jufqu'à ce que le bornage \& la mife en règle des forêts, qui font très-avancés, foient achevés ; fuivant les art. 9 tit. $2, \& 6$ tit. 3 de l'organifation foreftière, les infpecteurs, à compter du premier janvier 1797 , feront tenus de connoître les règles de larpentage; il n'exiftera plus d'arpenteurs à la charge de l'état; \& actuellement, vu le petit nombre de ceux qui font néceflaires, une fomme de 40,000 liv. eft plus que fuffifante pour levirs falaires. L'art. I I, au lieu de 757 mille livres, doit donc être réduit à

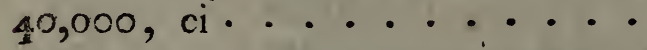

L'art. I2, pour les gages des gardes, eft de beaucoup exagéré; on trouvera facilement des gens aptes \& honnêtes qui

44,00001

2,0001

$947,500 \mathrm{~L}$

40,0001

$44,00000 \mathrm{I}$. 
D'autre part... ( II) . . 44,000001. s'en chargeront pour fix fols par arpent; cette dépenfe fera donc réduite

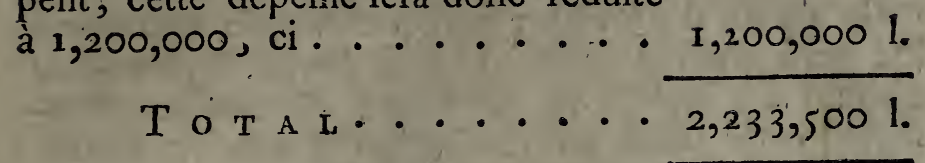

\section{R É S U M É.}

Commiffaire. .......

Bureaux. . . . . . .

Regiftres.............

Confervateurs, infpecteurs \& fuppléans .........

Arpenteurs. . . . . . . $\quad 40,000$

Gardes......... 1,200,000

$$
\text { E G A L I T É..... }
$$

Mais outre que, comme je l'ai démontré précédemment, ces frais feront dans cinq ans diminués de 40,000 livres, il y a un moyen jufte \& facile de réduire la dépense à la charge de l'état, en en faifant fupporter une partie aux municipalités propriétaires de forêts.

De tout temps, Meffieurs, les municipalités ont contribué aux honoraires de ceux qui adminiftroient leurs bois, \& il eft en effet de toute juftice que chacun participe aux frais de l'adminiftration, lorfqu'il en tire de l'avantage: or, les municipalités poffèdent $2,202,134$ arpens de forêts; en fuppofant que les 


\section{(12)}

quarts de réferve fubfiftent comme précédemment, il refte pour les coupes ordinaires I, 655,000 arpens qui aménagés à 25 ans de recrute, donneront 66 . mille arpens d'affouage: or, que chaque municipalité verfe dans la caiffe de fon difrict 3 livres par chacun des arpens qui lui feront délivrés annuellement, cette modique rétribution diminuera la dépenfe de la nation de 196,000 livres.

$S i$ les quarts de réferve fubfiftent, les 2- fols pour livre de ceux qui fe vendront annuellement, \& qui ont toujours été verfés dans les coffres de l'état, produiront au moins $50,000 \mathrm{l}$. par an ; la foible mais jufte contribution des municipalités, dans les frais de l'adminiftration foréftière', fera donc de 246,000 livres, qui, déduits des 2,233,500 livres gue j'ai prouvé être le plus haut taux auquel ils puifient s'élever, réduiront cette dépenfe à I,?87,500 livres, conféquemment très-près de moitié moins, que la fomme à laquelle on l'avoit arbitrairement portée; \& certes cette dépenfe n'eft point trop forie pour un revenu de 25 millions: l'Affemblée nationale laiffe fubfifter les adminiftrations des poftes \& meffageries, qui ne rapportent pas à beaucoup près autant, en proportion des frais qu'elles coûtent ; les frais de l'adminiftration des forêts feront encore réduits par la part 'qu'en fupporteront les propriétaires d'ufines à feu qui s'alimentent par des affectations en forêts nationales; ces propriétaires ont toujours payé les officiers de maîrifes pour les bois quils leur délivroient; ils verferont dans les caiffes de diftrict les fommes qu'ils donnoient à ces officiers.

Mais fi l'Affemblée nationale trouve encore que les finances del'état ne lui permettent pointide fupporter cette dépenfe ainfi réduite, il eft un moyen fimple $\&$ jufte de la faire fupporter entièrement par le luxe, 


\section{( ( I3)}

- fans qu'il en coûte rien à la claffe peu aifée des citoyens; ce moyen eft d'établir une taxe fur les cheminées en exemptant de cet impôt töut citoyen qui n'àra pour toute fa famille qu'un ou deux feux; de cette manière, les grands confommateurs de bois paieront feuls les frais d'une adminiftration dont le produit tourne principalement à leur commodité; ¿x les citoyens qui n'en confomment que l'indifpenfable pour leur chauffage \& la cuiffon de leurs alimens, feront indemnilés en partie du haut prix auquel ils font forcés d'acheter le bois.

Je paffe à la troifieme propofition des partifans de l'aliénation; elle confifie à dire que les fonctionnaires créés par la loi de l'organifation foreftière, étant en nombre très-inférieur à ceux des ci-devant maîtrifes, qui felon eux ne pouvoient fuffire à lcurs fonctions, à plus forte raifon, les nouveaux adminiftrateurs ne pourront-ils les remplir. On ajoute que leur refponfabilité fera illufoire comme ci-devant, parce qu'ils fe pafferont réciproquement leurs malverfations : il eft facile de faire tomber cette objection; fes auteurs ignorent ou feignent d'ignorer que les opérations de campagne, des ci-devant maitrifes, leur prenoient tout auplus deux mois par an, \& que pendant les dix autres mois, leurs fonctions fe bornoient au jugement des délits foreftiers \&à un très-léger travail de cabinet. Après cette obfervation préliminaire, il me fera facile de démontrer que les nouveaux fonctionnaires fuffiront, fans être chargés, à toutes les opérations gruriales.

Je dois à cet effet rappeler que dans la totalité des forêts nationales \& des municipalités quife portent àcinq millions cinq cents quatre-vingt-onze mille cing cents quatre-vingt-dix.fept arpens, le domaine en poffédoit neuf cents vingt-fix mille cinquante-quatre; les princes apanagés trois cents trente-fept mille neufcents fix, \& que 


\section{$(14)$}

les forêts engagées féportent à quarante-trois mille arpens; toutes ces forêts n'avoient point de quart de réferve, \&étoient divifées en coupes annuelles : mais lesci-devant corps eccléfraftiques \& les municipalités en poffédoient quatre millions dewx cents quatre-vingt - quatre mille trente arpens. Cette maffe, qui approche des quatrecinquièmes de toutes les forêts fur lefquelles les adminiftrateurs doivent opérer, n'eft point toute divifée en coupes annuelles; le quart en a été diftrait pour fubvenir aux befoins extraordinaires des corps auxquels il apparienoit; il s'exploite tout-au-plus une fois dans vingt - cinq ans : ce quart eft d'un million foixanteonze mille fept arpens; en le diftrayant des forêts deftinées aux coupes anvuelles, elles feront réduites à quatre millions cinq cents vingt mille cinq cents quatre-vingt-dix arpens. Divifez cette quantité par le nombre de vingt cinq, qui eft l'âge auquel feul en général on doive couper les bois, le quotient vous donnera cent quatre-vingt-deux mille deux cents trente arpens de coupe annuelle \& quelques fractions ; répartiffez enfuite ce quotient fur les trois cents trois infpecteurs, chacun d'eux n'aura que fix cents onze arpens \& demi \& quelques fractions à délivrer par année, ce qui ne fait pas deux arpens par jour; ôtez fix mois dans lefquels on n'opère pas dans les bois, la délivrance journalière des infpecteurs ne s'élèvera pas à quatre arpens : or, tous ceux qui connoiffent les opérations foreftières, favent qu'un infpecteur peut délivrer douze arpens par jour; il aura donc fuffifamment de temps pour la vifite des forêts de fon arrondiffement, qui ne fe porteront pas à dix-neuf mille arpens, pour la pour̀fuite des délits, la rédaction des procès-verbaux \& fa correfpondance avec fes fupérieurs. Au furplus, dans les cas urgens, il trouvera du fecours dans les fuppléans de fa divifion. 


\section{(15)}

Quant au danger que les fonctionnaires ne négligent leurs fonctions, il eft un moyen bien fimple d'y obvier; ils ne peuvent toucher leurs gages qu'en vertu de mandement des corps adminiftratifs : ces corps font chargés de les furveiller; qu'on les rende refponfables des mandemens qu'ils auront délivrés aux fonctionnaires négligens ou malverfateurs, \& l'on aura une certitude morale que l'argent de la nation ne fera délivré qu’à ceux qui auront fait leur devoir.

Sur le quatrièmè motif, les préopinans ont foutenu qu'en général les biens appartenans à une fociété étoient plus mal tenus que ceux des individus; ils en ont donné pour exemple les communes. Ils ont ajouté que les forêts des particuliers étoient d'un plus grand produit quie celles de la nation.

Je conviens avec eux de la première propofition; il eft de fait en général que les propriétés privées font mieux cultivées que les propriétés communes mais quant à la feconde propofition, elle eft démentie par l'expérience, \& je n'héfite pas de pofer en fait la propofition abfolument contraire.

Il eft depuis plufieurs fiècles tellement notoire quelês particuliers dégradent entièrement leurs bois; que lés lois foreftières, pour empêcher cet abus, ontété forcées de les foumettre à l'infpection des officiers royaux; \& malgré leur furveillance, à l'exception de quelques ci-devant corps religieux, qui adminiftroient leurs bois, en bons pères de famille, en général ceux des particuliers font les plus mal tenus du royaume.

En effet, outre que l'impatience de jouir ne leur permet pas d'attendre la maturité de leurs coupes, s'il y exiftoit un pied d'arbre beau \& fufceptible d'accroifement, il étoit le premier fous le tranchant de la coignée; s'ils fe conformoient à l'ordonnance pour le nombre des réferves, ils ne laiffoient que les arbres 


\section{( I 6)}

qu'ils avoient peu d'intérêt à abattre; fi quelques pères économes \& rangés avoient maintenu une bonne police dans leurs bois, les enfans moins aifés ou prodigues, détruifoient dans un jour le fruit de l'ordre ce leur père; \& comme de toutes les propriétés, il n'en eft point qui fe reproduife plus lentement que les forêts, la faute d'sn jour coûtoit un fiècle à reparer. Il eft donc de fait en général que, quo:que les forêts nationales ne foient point aufr bien tenues qu'clles pourroient l'être, ceilles des particuliers font dans un beaucoup plús mauvais état.

Sur le cinquième motif, je dirai qu'il n'eft nullement démontré que l'état de nos finances exige le facrifice denas forêts; il me paroît que l'inquiètude des comités des finances fur le paiement de la dette exigible leur a fait de beaucoup exagérer le paffif \& dininuer l'actif. Celte crainte fait honneur à la loyauté des membres; mais aucun d'eux ne fait à quel taux s'élevera la maffe des biens qui nous reftent à vendre; l'augmentation progreffive de leur prix doit relever leurs efpérances; \& il exifte d'ailleurs une infinité de domaines engagés ou aliénés dans lefquels la nation eft en droit die rentrer.

Les partifans de la vente do vent convenir que la vente, des forêts ne feroit indifpenfable, quautaght qu'elle fourniroit de quoi éteindre complétement notre paffif. Or, il eft certain : $1^{\circ}$. que pour lavoir sil fera éteint, il faut connoitre pofitivement en quoi il confifte, \& cette certitude ne peut - être acquife qu’à l'expiration du délai accordé aux créanciers de l'état pour produire leurs titres; or, il fe paffera près de deux ans, avant que ce terme ne foit expiré pour cenx qui habitent les ifies \& nos poffeffions au-delà du Cap-de-Bonne-Fípérance; jufqu'à cette date donc il eft impoffable de favoir le montant de notre pafif.

$$
2^{\circ} \text { Si }
$$




\section{( 17 )}

20. Si les calculs des comités des finances font juftes, il s'en faudra de beaucoup que l'aliénation des forêts fuffife pour éteindre le déficit; or y comme le projet des partifans de l'aliénation porte principalement fur la fuppofition que ce defficit fera éteint; le principe ceffant, la conféquence doit également tomber.

$3^{\circ}$. Outre qu'il y a une impolitique évidente d'expofer en vente une trop grande maffe de biens, parce que la multiplicité diminue la concurrence \& fait ba:ffer le prix du tout; quelle garantie peuvent, nous donner les partifans de l'aliénation, que cette maffe énorme, de biens trouvera des acquéreurs? Penfent-ils que la fortune des particuliers foit un fonds inépuifable ? N'eft-il pas clair que le hauffement énorme du prix de toutes les denrées la baiffe fenfiblement? $\mathrm{Ne}$ fentent-ils point que nos troubles, \& notre fituation incertaine vis-à-vis des étrangers, arrêteront néceffairement les ventes; \& que tant que la paix intérieure \& extérieure ne fera point folidement établie, les étrangers fe garderont bien de faire des acquifitions, dont ils ne trouveroient point une garantie fuffifante?

$4^{\circ}$. Il eft convenu que la maffe des affignats en circulation eft trop confidérable, \& il eft arrêté que de 1500 millions on la réduira à 900 . Or, en fuppofant que notre dette exigible foit auffi confidérable, que Meffieurs des finances le fuppofent, \& notre numéraire étant prefque entièrement difparu, jil eft clair que l'on ne pourra acquitter cette dette qu'en papier monnoie; on fera donc forcé de le multiplier au lieu de le reftreindre.

$5^{\circ}$. L'affemblée conftituante \& celle léginative, en mettant toute la dette publique faus la fauve-garde de la loyauté de la nation, n'en a pas excepté la dette cunftituée: or, il eft avoué que la nation, malgré que

Objerv. Jur l'alién. des forêts nat. 


\section{$(18)$}

for engàgemènt n'excepte rien, ne veut point rembour. fer cette derniêre ; il 'leeft également fuivant les comités des finances, que Paliénation des forêts ne fưffit pas pour éteindrè lâ dette exigible; it y aura done toujours des créaniciers de cette dernière claffe qui ne féront point acquittes; or, pourquioi tie lé feroienttils point comme les premiers liquidés? Leurs titres ne foht-ils pảs les mêmès', \& fi l'on regarde comme unéefpéce de banqueroute de ne point les défintéreffer tóuís, les derniers ne feront-ils pas fondés à fe plaintidre de ce que la nation ne leur a pas fait la mêne fuftice gh'd d'attites ģui n’ávoient pas plus dè privilège?

Wil réfulté de ces obfertations. que la vente des forêts 'rationales 'ne poutvant atteindre le but pour Tequel les partifans de laliénation la provóquent, if feroit auffimprudent quimpofitique de prendre cette méfuré.' Je parfe à l'établiffementit des propofitions que jai pofées'avant de difcuier Yés motifs des partifans de la vente.

- L'aliénation des forêts enl provógueroit la deffruction. Làtoi furr l'organifation fóreftière, fouftrait les bois des particuliers a toute infpection \& régime, elle leur en laifie la difpofition illimitée; ils font thes maitrés - de les effarter \& de les changer de nature; qui ne voit que tout acquéreur empreffé de fe libérer $\&$ de jouir, coúpera toute la fuperficie de fes bois? \& priverá le public de la reffource annuelle qu'it $y$. trouvoit, loifqu'ils étoient divifés en coupes réglées. On répondrà que l'on peut parer à cèt inconvenient én-limitant la liberté des acquéreurs des forêts; más outre que cette limitation eft contraire à l'efprit de' la conftitution, it eft fénfible que plus vous reftreindrez la jouiffance, moins vous trouverez d'acquéreturs ; il en réfultera que vos forêts pafferont prefqué exclufivement dans les inains de monopoleurs a fecula-

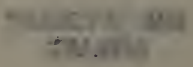


tions, qui, à défaut de concurrence, les acheteront à vil prix.

L'aliénation feroit deftructive du commerce, dans une portion confidérable de la France

Les départemens de la Meurthe, Meufe, Mofelle, Vofges, Doubs, Jura, Haute-Saone, à raifon de l'humidité du fol \& de la graiffe de leurs pâturages, n'ont que des laines très-groffières; la même caufe \& la froideur du climat, leur interdit l'éducation des vers à foie, \& ne leur donne que des lins \& chanvres de la dernière qualité ; ces départemens n'ont donc point de manufactures; ils font forcés de tirer de l'intérieur du royaume les foieries, draperies \& toiles. II en eft à-peu-près de même des départemens du haut \& bas Rhin. Ce commerce fait fortir de tous un numéraire immenfe.

La nature leur a donné en dédommagement des fources falées \& des mines de fer; l'induftrie des habitans, qui ne pouvoit foutenir la concurrence des autres fabriques du royaume, s'eft portée vers: lexploitation des mines \& des autres ufines à feu ; dépourvus de foffiles combuftibles, mais riches en forêts, dont la quantité, dans l'ancienne province de Lorraine feule, eft t̀-peu près d'un quart de toutes celles du royaume, les habisans ont établi \& contruit des falines, des forges, fonderies, ferblanqueries, des, verreries \& faianceries; le produit de ces manufactures, verfé chez l'étranger \& dans l'intérieur de la France, ramèrie une partie des fommes que l'importation des foieries, draperies \& toiles en a tirées.

La majeure partie de ces ufines a une affectation emphytéotique dans les forêts nationales; tous les entrepreneurs n'ont conftrwit que dans l'affurance qu'ils auroient les bois à bas prix; - fi la nation retire les forêts \& les met en vente, outie qu'clle fera forcée B 2 


\section{(20)}

d'indemnifer les emphytéótes de la non-jouiffance de leurs baux, ce qui égalera peut-être le prix de la vente des forêts, toutes les ufines, à défaut d'alimens, ou forcées de les acheter au prix que les acquéreurs feront les maîtres de tenir le bois, tomberont d'elles-mêmes; dix mille ouvriers, habitués dès l'enfance au travail de ces uínes, refteront fans reffource, feront plongés dans l'extrême misère, \& la France perdra une branche de commerce florifante.

Je dònnerai un échantillon de cette perte. Les falines de la Metirthe ont quarante-cinq mille arpens de bois d'affectation qui leur donnent trente-fix mille cordes de bois d'affouage annuel. Le prix de la corde de bois, fi elle fe vendoit, ne fe porteroit point à 6 liv. ce quỉ pour les trente-fix mille cordes produiroit au plus 2 I 6,000 1. Eh bien! ces trente-fix mille cordes de bois converties en fel, outre qu'elles font vivre plus de mille employếs \& ouvriers, vaudront à la nation deux millions de revenu net, très-fufceptible d'augmentation; le fait fera démontré dans le rapport fur les falines de la Meurthe, dont s'occupe le comité des Domaines.

L'aliénation des forêts feroit deftructive de la population \& de l'agriculture, dans les départemens de la Mofelle, des Vofges \& de la Meurthe; les forêts de ces départemens font en grande partie accenfées aux munic palités de campagne', qui ont droit d'y prendré le bois de conftruction \& de maronage, le bois mort $\&$ mort bois, \& la graffe $\&$ vaine pâture.

C'eft fur la foi de cet accenfement que les municipalités fe font établies, on ne peut les en priver fans indemnité; fous ce point-de-vue la vente des forêts feroit done peu profitable, mais elle feroit vraiement défaftreufe fous un autre afpect; dans les Vofges \& la partie allemande des départemens de la Mofelle 


\section{(21)}

\& de la Meurthe, il exifte très-peu de prairies, l'habitant n'a de reffource pour fon bétail que la vaine pâture dans les forêts; fi on l'en prive, l'agriculture tombe, \& avec elle les moyens de fubfifter.

Mais l'agriculture effuieroit encore une autre perte très-fenfible par l'aliénation des forêts : les départemens compris dans les ci-devant provinces de - Lorraine, Trois-Évêchés \& Franche-Comté, ont en général un fol.de terre forte \& compacte, les chemins vicinaux y font très-mauvais \& prelque impraticables; il en réfulte que les inftrumens aratoires \& de tranfport fe rompent très-fréquemment ; le bois de charonnage doit donc être commun \& à bon prix dans ces départemens; \& il eft évident que par l'aliénation des forêts il fe portera à un prix auquels les laboureurs ne pourront atteindre: ainfi une portion confidérable des plus productives de la France qui alimente la majeure partie des départemens du midi, deviendra inculte. Il refte deux confidérations locales qui démontrent que l'aliénation des forêts entraîneroit la dépopulation de ces départemens, ainfi que de ceux du haut \& bas-Rhin; dans une grande partie de ces cantons il n'exifte ni moëlon, ni taille, ni ardoifière, ni argile propre à la tuile; les habitans font forcés à conftruire \& couvrir la totalité de leurs édifices en bois; la froideur du climat, occafionnée par le voifinage des montagnes \& la fréquence des étangs \& marais, prolonge l'hiver l'efpace de fix mois, pendant lefquels il eft impoffible de fe paffer de feu; la nature ayant refufé à ces départemens des foffiles combuftibles, ils font forcés à employer le bois pour leur chauffage. Les acquéreurs qui n'ignoreront point cette circonffance, convaincus que les bois de conftruction $\&$ de chauffage font de première $\&$ abfolue néceffité, exerceront un monopole ruireux, \& les habitans ne 


\section{$(22)$}

pouvant plus ni le loger ni fe chauffer, feront réduits a s'expatrier, \& la nation perdra un million de citoyens laborieux.

Ce tableau, qui n’eft nullement exagéré, doit faire fentir combien l'aliénation feroit difficile à exécuter; on conçoit en effet à quels excès le défefpoir peut porter une fi grande portion de citovens forcés à quitter les champs qui les ont yu naître, \& à abandonner. une patrie que la conftitution leur faifoit ardemment chérir; vous les obligerez à fe jeter dans les bras des émigrés , \& vos départemens de la frontière la plus expofée deviendront, par votre fait feul, le foyer de la contre-révolution.

La vente des forêts feroit très-préjudiciable à la marine. Toutes les rivières navigables de l'intérieur affluent à nos ports de l'océan \& de la méditerranée : il eft donc on ne peut plus facile d'y faire parvenir, foit en grume, foit en madriers, les bois de conftruction, percrus dans les forêts de l'intérieur. Dans la guerre maritime de $175^{8}$, où les Anglais, après avoir détruit nos forces navales, étoient maitres de la mer, il nous étoit impoffible de tirer des bois de l'étranger pour remplacer nos vaiffeaux pris. Notre commerce fans protection, devint la proie de nos ennemis, \& nos négocians fe fentent encore de cette perte.

Cet événement ne peut-il pas fe renouveller, \& nous ôterons-nous, en aliénant nos forêts, la faculté de conftruire avec les bois de notre cru? Non, les générations actuelles \& futures n'auront point à reprocher à la première Affemblée légillative, de lui avoir fait cette plaie irréparable.

On objectera que nos forêts ne fuffifent pas à beaucoup près pour l'entretien de notre marine, qu'elles ne fourniffent point de mâture. Je conviens qu'à l'exception de la Corie, nos autres provinces ne fournif- 


\section{$(23)$}

fent point de mâts, tels que notre marine en emploie? mais les móntagnes des -Voges ont les plus belles fapinieres; perfonne n'igniore que les flottes ruffes font entièrement conftruites de cette efpèce de bois; \& pourquioi ne tirerións fious pas le même parti dé nos productiors naturelles. Les départemens de la Meurthe, Meufe \& Mofelle', produifent une quantite confidérable de chêne de la plus belle \& meilleur efpèce; il y a trente ans que dans les feulec forêts du diftrict de Bitche, on vendit cinquante mille pieds d'arbres chênes, \& elles contiennent encore une infinité d'arbres de la même efpèce ; les hollandais, qui furent adjudicataires de cette vente, dont ils firent paffer le bois de conftruction dans leurs ports, par la Sarre, la Mofelle \& le Rhin, continuent ce commerce, ramenent ce bois en France, où ils le vendent au poids de l'or; jufques à quand la Nation fouffrira t-elle que des étrangers s'enrichiffent ainfi de nos propres productions? Le miniftère de Louis XV avoit effayé de le leur enlever, en prohibant momentanément la fortie des bois du royaume; mais fon infouciance ne lui ayant fait prendre aucun moyen pour les employer à l'avantage de la Nation, le prix des ventes baiffa, \& la prohibition fut révoquée ; il eft cependant très facile de nous àpproprier ce commerce; fi l'on ne joint point, par un canal de navigation, la Meufe \& la Mofelle à la Marne, comme la chofé eft très-poffible, que l'on établiffe des commiffaires à réfidence dans les quatre départemens de la ci-devant Lorraine; qu'ils foient tenus de parcourir les coupes annuelles, \& de réferver pour la marine, les bois qui lui conviennent, qu'on les faffe flotter dans nos ports par la même voie que les hollandais, \&notre marine aura à bon prix, une quantité de bois de mreitleur effence que ceux que lon tire du Nord. Je me réfume 
' je dis, j'ai prouvé que l'aliénation de nos forêts feroit deftructive des bois, d'une branche précieufe de notre commerce, de l'agriculture \& de la population dans une portion confidérable de l'empire, trèspréjudiciable à notre marine, peu profitable à l'Etat \& peut-être impolfible à exécuter. Je conclus en conléquence à adopter le projet de décret du comité.

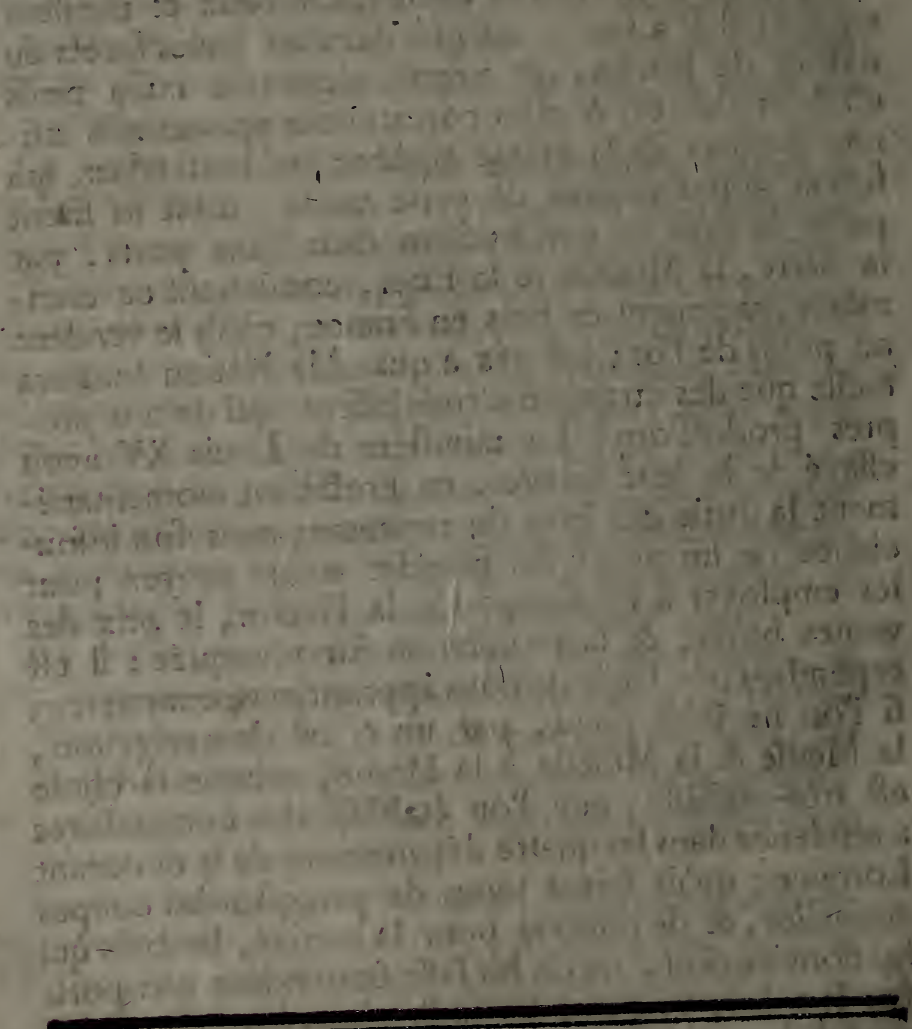

DE LIMPRIMERIE NATIONALE. $5 \ldots$ 